\title{
SERODIAGNOSIS OF TOXOPLASMA GONDII, ASSOCIATED RISK FACTORS IN DOMESTICATED CATS: PREVENTING ZOONOSIS IN HUMANS AND IMPLICATIONS FOR LIVESTOCK EXTENSION
}

\author{
aMazhar Abbas, aAmar Nasir, aMuhammad Kashif, bashif Hussain*, bNaheeed Bano, buhammad A. Raza, \\ bWaqar Zaib, cRao Z. Abbas, dMuhammad Nadeem, bRana W. Akhtar, cTean Zaheer, bMuhammad U. Waqas, \\ cZurisha Rani, eArbab Sikandar, bAsghar Abbas, bAtif Rehman, bHafeez R. Khera \\ ${ }^{a}$ Department of Clinical Sciences, Sub-campus Jhang, University of Veterinary and Animal Sciences, Lahore, Pakistan. \\ ${ }^{b}$ Faculty of Veterinary and Animal Sciences, MNS-University of Agriculture Multan, Pakistan. \\ c Department of Parasitology, University of Agriculture Faisalabad, Pakistan. \\ d PMAS-Arid Agriculture University Rawalpindi, Subcampus, Khushab. Pakistan. \\ e Department of Basic Sciences, Sub-campus Jhang, University of Veterinary and Animal Sciences, Lahore Pakistan.
}

ARTICLE INFO

\section{Article History}

Received: January 18, 2020

Revised: March 12, 2020

Accepted: April 27, 2020

\section{Keywords}

Toxoplasmosis

Seroprevalence

Immunity

Zoonosis

Livestock extension
A B S T R A C T

Toxoplasmosis, an important zoonotic parasitic disease-causing infection in humans and contaminating the environment. Cats are the sole source of spreading infection. Current study was designed to serodiagnoses in the flooded area, Jhang, Pakistan. Using rapid identification pen side strip test 316 pet cats were examined at CVAS, Jhang and other private clinics in that area. Rapid ID Chromatographic immune assay was performed to detect the antibodies for Feline toxoplasma IgM and IgG in serum. Related associated risk factors like breed, sex, contact with other livestock animals, deworming, location type (urban or rural area), diet (nature of food either cooked or uncooked meat), and outdoor access for wandering were also interviewed by owner. Total seroprevalence of cats was 10.4\% (33/316). IgG antibodies were found 9.09 $(29 / 316)$ while IgM antibodies were $2.21 \%$ (7/316) in cats. Seroprevalence was significantly high in cats older than one year. No significant difference was recorded between males and females. Cats from peri urban areas showed higher prevalence. Cats having access to outside, contact with other animals and eating uncooked food showed high seroprevalence. The current study confirms that Toxoplasma gondii is widespread in pet animals in district Jhang, Pakistan. This study urges pets lower to be aware about the Toxoplasmosis diseases. Whereas, the livestock extension sector should disseminate the precautionary awareness among the public.

Corresponding Author: Kashif Hussain

Email: kashifhussain1167@gmail.com

(C) The Author(s) 2021.

\section{INTRODUCTION}

Parasitic diseases cause serious threat to humans and animals all over the world (Abdel-Sattar et al. 2019; Hassan et al. 2020; Tammam et al. 2020). Among parasitic diseases, Toxoplasmosis, an infectious parasitic disease, is caused by an obligatory intracellular protozoon Toxoplasma gondii belonging to phylum apicomplexan. It is of great zoonotic importance, infecting humans and other warm-blooded animals which are intermediate host (Awad and Barakat, 2019). It is distributed worldwide, infecting one third of world's human population and contaminating the environment 
with resistant oocytes (Zhang et al., 2016; Dubey, 2001; Okewole and Akpan, 2002). Cat either wild or domesticated is the definite host for shedding of oocytes in feces. The transmission of infection is possible through both vertical (via placenta) and horizontal (fecal oral route, ingestion of uncooked meat containing oocysts and via carnivorism route). Cats (Felis catus) as pets, are the sole source of infection transmission to human community from contaminated environment through feces and mechanically by flies, cockroaches, dung beetles, and earthworms (Dubey, 2004). Toxoplasmosis is also known as water-borne disease as spreading through sewage water and infecting humans in urban areas where it is difficult to treat water with process of chlorination and decantation (Meireles et al., 2004). Serious losses are observed in different species of livestock and in pets(dogs and cats) of different ages due to mortality, embryonic death and resorption, fetal mortality and mummification, abortion, stillbirth, neonatal death and immunosuppression (Dubey, 2002). While in humans, it causes asymptomatic disease in adults, and the infection depends on the stage of pregnancy, developmental abnormalities and blindness in fetus that may arise from infected mother (Ahmad et al., 2019). Oocysts are more dangerous to cause disease than cysts (Dubey, 2009). But the major losses are socioeconomics as long period treatment and children's care (Roberts et al., 1994). Direct smear method is used to diagnose this disease through the feces concentration but it is less sensitive and difficult to differentiate Hammondiahammondioocysts (Györke et al., 2011). Antigen is detected through molecular technique by PCR but it is time consuming. In current study serologically Abs has been diagnosed in infected cats which is rapid ID test and less expensive as well.

\section{MATERIALS AND METHODS}

\section{Study Area}

This research study was approved by the Departmental Research Ethics Committee and was conducted following the ethical guidelines. This study was conducted in the district Jhang of the Punjab province. Jhang district, land of two rivers Jehlum and Chenab, is situated in the central Punjab, Pakistan. Population according to census 2017, is 2835000 at the area of $33097 \mathrm{~km}$.

The climate of district is mild warm and temperate. More rain falls in summer than the winter. Average temperature is $21.35^{\circ} \mathrm{C}\left(32.5-10.2^{\circ} \mathrm{C}\right)$ annually while the annual rain fall is recorded $679 \mathrm{~mm}$. Maximum rain of $166 \mathrm{~mm}$ is recorded in August and minimum rain of $9 \mathrm{~mm}$ in November.

\section{Study Animals and Sampling}

316 cats of both sex, and different breeds and ages were examined at the outdoor clinic of College of Veterinary and Animals Sciences during the period of 2019. Clinical signs and symptoms of toxoplasmosis were observed including fever, loss of appetite, and lethargy. Blood samples were collected from the saphenous and jugularvein of examined pet cats with minimal doses of sedation according to Animal Welfare protocols (Ahmad et al., 2014).Serum from collected blood was separated by centrifugation at $3000 \mathrm{rpm}$ for 1 minute and stored at $-20^{\circ} \mathrm{C}$ until analysis was performed.

\section{Epidemiological Information}

A questionaries' was designed to obtain the epidemiological data regarding age, breed, sex, contact with livestock animals, deworming, location type (urban or rural area), diet (nature of food either cooked or uncooked meat) and outdoor access for wandering (Sedlak and Bartova, 2006) and direct contact with soil.

\section{Serodiagnosis}

Rapid ID Chromatographic immune assay was performed to detect the antibodies for Feline toxoplasma IgM and IgG in serum(Luo et al., 2018). Single step pen side test kit was used for antibodies detection against Feline toxoplasma, supplied by Bionote, Korea. Results were interpreted according to the manufacturer's instructions.

\section{Statistical Analysis}

Univariate and Pearson Chi-Square Tests were used to find out the relationship between serodiagnosis of toxoplasmosis and various risk factors (like sex, age, area, diet etc.) by using IBM SPSS Statistics 21. Different aspects of study were found like odd ratio, percentage and prevalence. Results were considered significant at $\mathrm{P}<0.05$.

\section{RESULTS}

The results obtained via rapid ID chromatographic assay are shown in the (Table 1). A total 316 samples were collected, out of which 33 were positive and 283 were 
negative. Four study groups were made for serological interpretation of IgM and IgG antibodies. Each group has different percentage of total number of examined cats. Seroprevalence for first group [IgG (-ev) IgM (-ve)] $89.6 \%$, for second group [IgG (+ev) IgM (-ve)]8.22\%, for third group [IgG (-ev) IgM (+ve)] $1.27 \%$ and fourth last group [IgG (+) and IgM (+)]0.94\% were found. A total seroprevalence of $\operatorname{IgG}$ and $\operatorname{IgM}$ are 9.09 and 2.21 respectively. But overall prevalence of $T$. gondii in domesticated cats was $10.4 \%$. Here it was seen that percentage of negative samples were high and low for positive.

Table 1. Number and percentage of IgM and IgG positive domesticated cats.

\begin{tabular}{lcc}
\hline \multirow{2}{*}{ Sero-group } & \multicolumn{2}{c}{ Cats } \\
\cline { 2 - 3 } & $\mathrm{N}$ & $\%$ \\
\hline IgG (-) and IgM (-) & 283 & 89.6 \\
\hline IgG (+) and IgM (-) & 26 & 8.22 \\
\hline IgG (-) and IgM (+) & 4 & 01.27 \\
\hline IgG (+) and IgM (+) & 3 & 0.94 \\
\hline Total IgG (+) & 29 & 9.09 \\
\hline Total IgM (+) & 7 & 2.21 \\
\hline Overall Positives & 33 & 10.4 \\
\hline Total Samples & 316 & -- \\
\hline
\end{tabular}

Related associated risk factors were observed as shown in (Table 2). It was seen that the cats of age more than one year, living in the peri urban areas and access to outside were more infected with $T$. gondii10.92\%, $22.35 \%$ and 40 , respectively. No significant difference was observed in the prevalence of both sexes $(\mathrm{P}>0.05$, $\left.\chi^{2}=1.918\right)$. Seroprevalence of uncooked meat $(12.50 \%)$ was higher than cooked (5.43\%) $\left(\mathrm{P}>0.05, \chi^{2}=2.481\right)$. Also, non-dewormed cats and contact with other animals showed high seroprevalence $\left(\mathrm{P}>0.05, \chi^{2}=2.11\right)$. Soil exposure to pet cats is another factor enhancing the chances of infection. Overall prevalence $(10.4 \%)$ of the current study shows that cats of District Jhang are exposed to T. gondii.

\section{DISCUSSION}

Parasitic diseases are continuously affecting both humans and Livestock population of both developing and advanced countries (Ali et al., 2020; Li et al., 2020). Toxoplasmosis, a common disease of cats, is difficult to diagnose upon its clinical signs Diagnosis is necessary for prevention and treatment of infection, and plays important role in transmission to human and contaminating the environment with oocytes. Cats are the definite hosts for shedding of oocytes in feces and cannot be diagnosed as eggs of other parasites are similar (Awad and Barakat, 2019). For rapid and accurate diagnosis, antibodies plays a major role in serological detection either acute or chronic form (Awad and Barakat, 2019). Detection of two types of antibodies IgG and IgM confirms T. gondii which is more reliable as compared to another pen side test (Luo et al., 2018; Dubey et al., 2003). For more confirmation tissue sampling is required for which killing of animal is required (Ahmad et al., 2014).

Epidemiological studies on prevalence of T. gondii in cat have been conducted in many parts of world. It occurs worldwide including Pakistan contaminating France (Afonso et al., 2006), Brazil (Pena et al., 2006), Czech Republic (Sedlak and Bartova, 2006), China (Zhang et al., 2009), and Iran (Raeghi et al., 2011). In Pakistan, Ahmad et al. (2001) and Ahmad et al. (2014) found 60\% in Faisalabad and $26.43 \%$ prevalence in subtropical Arid parts in cats, respectively. The current study was designed to check the prevalence and risk factors favoring $T$. gondii in domesticated cats brought at College clinic for checkup and other veterinary clinics in District Jhang. For rapid identification serological test four groups were made and results were obtained.

Cats living in urban community were more affected than cats living alone (peri urban), and this factor is of vital in transmission of disease to humans due to congested population. Access to outside and soil exposure in rural and preurban areas are more as compared to urban which is the source of environmental contamination having oocytes. Cats either pets or stray play with soil get more chances of infection agreed by the Györke et al. (2011). One more thing, stray felids and livestock 
populations are high in rural areas so, there is a high risk of getting disease. Also other cats in same area may be susceptible to toxoplasmosis as described earlier by Lopes et al. (2008) and Györke et al. (2011).

Table 2. Factors associated with T. gondii in cats.

\begin{tabular}{llccccc}
\hline Factors & Categories & $\mathrm{N}$ & $\mathrm{C}+$ ) & $\%$ & $\mathrm{P}$ & Odd ratio (CI 95\%) \\
\hline Area & Urban & 231 & 14 & 6.06 & .011 & Reference \\
& Peri urban & 85 & 19 & 22.35 & & $2.536(1.214-5.296)$ \\
\hline Access to outside & Yes & 45 & 18 & 40 & .000 & Reference \\
& No & 271 & 15 & 5.53 & & $8.243(3.766-18.043)$ \\
\hline Age & $>1$ year & 238 & 26 & 10.92 & .950 & Reference \\
& $<1$ year & 78 & 07 & 8.97 & & $0.974(0.420-2.257)$ \\
\hline Sex & Female & 146 & 17 & 11.64 & .166 & Reference \\
& Male & 170 & 16 & 9.41 & & $1.667(0.804-3.456)$ \\
\hline Diet & Uncooked & 224 & 28 & 12.5 & .115 & Reference \\
& Cooked & 92 & 05 & 5.43 & & $0.530(0.238-1.180)$ \\
\hline Deworming & Yes & 226 & 11 & 4.86 & .000 & Reference \\
& No & 90 & 22 & 24.44 & & $0.135(0.0610-0.298)$ \\
\hline Contact with other animals & Yes & 264 & 29 & 11.00 & .000 & Reference \\
& No & 52 & 4 & 8.00 & & $0.166(0.069-0.398)$ \\
\hline Soil exposure & Yes & 129 & 20 & 15.50 & .011 & Reference \\
& No & 187 & 13 & 6.95 & & $0.344(0.754-0.291)$ \\
\hline & & & & & &
\end{tabular}

As the age is concerned, more in geriatric cats of age more than one year or above $(10.92 \%)$, they have more exposure of $T$. gondii in their life than younger one (8.97\%). Infected intermediate hosts (rat, birds and small mammals) may be the carrier of tissue cyst, and cats (feral and stray) get infection during carnivorism. High prevalence has been already determined by Pena et al. (2006) based on including this factor as well. According to current study high seroprevalence in the cats, fed with uncooked meet and having outdoor access outdoor, were observed (Gauss et al., 2003). According to current study it was found that there is no significance difference between seroprevalence of male and female as described by (Lopes et al., 2008). Significant differences were found in the area, access to outside, age and diet as earlier reported by Awad et al. (2018).

\section{CONCLUSION}

Toxoplasmosis is a zoonotic disease, known as water borne zoonosis. It is the source of environmental contamination to cause the infection in humans. It is impossible to diagnose upon clinical examination so we move to serological techniques which are economic and less time consuming as compare to other molecular techniques. Here, rapid identification is the sole source which confirms antibodies against toxoplasma at the spot in domesticated pet cats. This study confirms that the cats were the source of zoonosis. The current study further confirms that Toxoplasma gondii is widespread in pet animals in district Jhang, Pakistan. This study urges pets lower to be aware about the Toxoplasmosis diseases. Whereas, the livestock extension sector should disseminate the precautionary awareness among the public. The livestock extension department should critically examine this vulnerability of community, should arrange different awareness creating activity among the public in order to prevent from the zoonosis.

\section{REFERENCES}

Afonso, E., P. Thulliez and E. Gilot-Fromont. 2006. Transmission of Toxoplasma gondii in an urban population of domestic cats (Felis catus). International journal for parasitology, 36: 137382.

Ahmad, F., A. Maqbool, A. Mahfooz and S. Hayat. 2001. Serological survey of Toxoplasma gondii in dogs and cats. Pakistan Veterinary Journal, 21: 31-35.

Ahmad, N., H. Ahmed, S. Irum and M. Qayyum. 2014. Seroprevalence of IgG and IgM antibodies and associated risk factors for toxoplasmosis in cats 
and dogs from sub-tropical arid parts of Pakistan. Tropical biomedicine, 31: 777-84.

Ahmad, N., I. A. Khan, Z. Iqbal, A. A. Naseem, A. R. Kayani, K. Afshan and M. Qayyum. 2019. Seroepidemiology of Toxoplasmosis in Human Population with Reference to Its Zoonotic Potential in Sub-Tropical Areas of Pakistan. Pakistan Veterinary Journal 39: 211-15.

Ali, S., Ijaz M, Ahmed A, Aziz M.U, Naveed M, Javed M.U, Nawab Y, Ghumman N.Z and Ghaffar A. 2020. Prevalence and associated risk factors of bovine babesiosis in Lahore, Pakistan. Agrobiological Records, 2: 17-23.

Awad, R. and A. Barakat. 2019. Serological Diagnosis of Toxoplasmosis in Household Cats in Egypt. Egypt Journal of Veterinary Sciences, 50: 57-63.

Awad, R. A., W. K. Khalil and A. G. Attallah. 2018. Epidemiology and diagnosis of feline panleukopenia virus in Egypt: Clinical and molecular diagnosis in cats. Veterinary World, 11: 578.

Dubey, J. 2001. Oocyst shedding by cats fed isolated bradyzoites and comparison of infectivity of bradyzoites of the VEG strain Toxoplasma gondii to cats and mice. Journal of Parasitology, 87: 215-19.

Dubey, J., R. Zarnke, N. Thomas, S. Wong, W. Van Bonn, M. Briggs, J. Davis, R. Ewing, M. Mense and 0. Kwok. 2003. Toxoplasma gondii, Neospora caninum, Sarcocystis neurona, and Sarcocystis canis-like infections in marine mammals. Veterinary parasitology, 116: 275-96.

Dubey, J. P. 2002. Tachyzoite-induced life cycle of Toxoplasma gondii in cats. Journal of Parasitology, 88: 713-17.

Dubey, J. P. 2004. Toxoplasmosis-a waterborne zoonosis. Veterinary parasitology, 126: 57-72.

Dubey, J. P. 2009. History of the discovery of the life cycle of Toxoplasma gondii. International journal for parasitology, 39: 877-82.

Gauss, C. B. L., S. Almería, A. Ortuño, F. Garcia and J. Dubey. 2003. Seroprevalence of Toxoplasma gondii antibodies in domestic cats from Barcelona, Spain. Journal of Parasitology, 89: 1067-68.

Györke, A., M. Opsteegh, V. Mircean, A. Iovu and V. Cozma. 2011. Toxoplasma gondii in Romanian household cats: evaluation of serological tests, epidemiology and risk factors. Preventive Veterinary Medicine, 102: 321-28.

Li, K., Luo H, Mehmood K, Shahzad M and Li J. 2020. Exploring the potential parasitic pathogens causing diarrheal death to yak calves with bloody excrement through high-throughput sequencing. Agrobiological Records, 1: 1-5.

Lopes, A. P., L. Cardoso and M. Rodrigues. 2008. Serological survey of Toxoplasma gondii infection in domestic cats from northeastern Portugal. Veterinary parasitology, 155: 184-89.

Luo, J., H. Sun, X. Zhao, S. Wang, X. Zhuo, Y. Yang, X. Chen, C. Yao and A. Du. 2018. Development of an immunochromatographic test based on monoclonal antibodies against surface antigen 3 (TgSAG3) for rapid detection of Toxoplasma gondii. Veterinary parasitology, 252: 52-57.

Meireles, L., A. Galisteo Jr, E. Pompeu and H. Andrade Jr. 2004. Toxoplasma gondii spreading in an urban area evaluated by seroprevalence in free-living cats and dogs. Tropical medicine \& international health, 9: 876-81.

Okewole, E. and M. Akpan. 2002. Clinical feline toxoplasmosis: parasitological, haematological and serological findings in retroviral infected and uninfected cats. Veterinarski arhiv, 72: 6779.

Pena, H. F. d. J., R. M. Soares, M. Amaku, J. Dubey and S. M. Gennari. 2006. Toxoplasma gondii infection in cats from Sao Paulo state, Brazil: seroprevalence, oocyst shedding, isolation in mice, and biologic and molecular characterization. Research in veterinary science, 81: 58-67.

Raeghi, S., S. Sedeghi and S. Sedeghi. 2011. Prevalence of Toxoplasma gondii antibodies in cats in Urmia, northwest of Iran. Journal of Animal and Plant Science, 21: 132-34.

Roberts, T., K. D. Murrell and S. Marks. 1994. Economic losses caused by foodborne parasitic diseases. Parasitology today, 10: 419-23.

Sedlak, K. and E. Bartova. 2006. The prevalence of Toxoplasma gondii IgM and IgG antibodies in dogs and cats from the Czech Republic. Veterinární medicína, 51: 555.

Zhang, H., D. Zhou, P. Zhou, Z. Lun, X. Chen, R. Lin, Z. Yuan and X. Zhu. 2009. Seroprevalence of Toxoplasma gondii infection in stray and household cats in 
Guangzhou, China. Zoonoses and public health, 56: 502-05.

Zhang, X.-X., W. Cong, J.-G. Ma, Z.-L. Lou, Q. Zhao, Q.-F. Meng, A.-D. Qian and X.-Q. Zhu. 2016. First genetic characterization of Toxoplasma gondii infection in Arctic foxes (Vulpes lagopus) in China. Infection, Genetics and Evolution, 44: 127-29. 